\title{
Making data accessible: lessons learned from computational reproducibility of impact evaluations
}

Neeta Goel ${ }^{a}$, Sayak Khatua ${ }^{b *}$, Marie Gaarder $^{b}$

${ }^{a}$ Bill and Melinda Gates Foundation

b International Initiative for Impact Evaluation (3ie), New Delhi, India

*Corresponding author: skhatua@3ieimpact.org

This version: January 30, 2021 


\section{Introduction}

To what extent are third party verifications of data and statistical code successful in reproducing reported research results? While the reproducibility of findings is an essential step to establish confidence in findings, there are indications that this is less common than one might believe. Originally noted in the psychology literature (Open Science Collaboration 2015), the 'replication crisis' (as it has come to be known) appears to be a cross-disciplinary challenge (Gertler, Galiani, and Romero 2018; Ioannidis 2005; Simmons, Nelson, and Simonsohn 2011; Humphreys, De la Sierra, and Van der Windt 2013). On the one hand, this suggests we may need to be more cautious about our confidence in scientific findings; on the other, these attempts to reproduce findings are also viewed as a best practice of good science (Loken 2019). The growing awareness that non-reproducible findings could most likely be wrong has prompted publishers and funders of scientific studies to review their quality assurance processes such as AEA's Data and code policies guidance ${ }^{1}$. But, while this has led to a call for more reproductions, in practice there are few incentives for doing so. Challenges include the unavailability of complete materials (datasets and code), stonewalling or hostility from original research team members when reproductions fail, and the preference of journals to publish 'original' research rather than reproductions of original research findings (Galiani, Gertler, and Romero 2017).

\footnotetext{
${ }^{1}$ Can be accessed at https://www.aeaweb.org/journals/data
} 
Research studies that are reproducible are particularly important in low and middle income-countries, where there is an emphasis on developing interventions and policies that are grounded in rigorous evidence (Khatua 2018). Given the limited resources available to tackle large scale challenges, it is imperative to ensure policymaking and programming draw upon lessons learned from evaluations of development interventions. But, given the replication crisis, how reliable is this evidence? While the literature on reproducibility of findings in the international development sector is sparse, the results are telling (International Initiative for Impact Evaluation(3ie), "Replication Studies", 2018). Most recently, Wood, Muller, and Brown (2018) find that of the 109 published impact evaluations in their sample, only results from 27 studies could be reproduced.

In its role as a producer of evidence in the Global South, the International Initiative for Impact Evaluation (3ie $)^{2}$ funds, quality assures and synthesizes impact evaluations of development interventions and policies in low- and middle-income countries. Since its inception in 2008, 3ie has been committed to providing open access to all 3ie-funded products (impact evaluation reports, systematic review reports, briefs), related data and code files once the studies are completed. Recognizing that the evaluation data could be useful to other researchers, we mandated the submission of datasets (and in later years, analysis code as well) which were uploaded to the Harvard Dataverse for public

\footnotetext{
${ }^{2}$ Available from: https://www.3ieimpact.org/
} 
access. However, not all evaluation teams fulfilled these requirements despite multiple rounds of follow-up.

The growing concern around the reproducibility of scientific studies prompted us to reflect on the reliability of the evidence that we funded. To ascertain this, we worked on a project to computationally reproduce published evaluation results using the original data and analysis code submitted by evaluation teams. We present the results of this exercise in this paper. Our primary finding is that we are able to reproduce findings from three-fourths of the 133 studies in our sample. This success rate is notably higher than those noted in similar literature. This can be partly attributed to the nature of our sample and partly to the stringent payment-linked measures (both described later in this paper) that 3ie adopted during the course of this study. Due to these reasons, we share these results purely as a descriptive study, and do not make any claims to this study's generalizability to other contexts. In our view, these results help to emphasize the role donor organizations can play in quality assuring results and contributing to a body of evidence that the development sector can be confident about.

In the next section, we describe our methodology and sample, and our findings in the subsequent section. Finally, we offer a few lessons learned which may be useful to researchers and other donor organizations.

\section{Methodology}

Since its inception in 2008, 3ie has been committed to open access to 3ie-funded publications and transparency all research practices in 3ie. In order to define our vision, and emphasize our commitment to transparent research procedures, we released our 
Research Transparency policy in 2018 ((3ie, "Research Transparency Policy", 2018). As a donor organization, the policy articulated our vision to ensure that the evidence we funded was open and accessible to all. It presented existing and proposed measures that would help us move in this direction. One of these measures was the decision that final grant payments would only be made after the evaluation findings (as laid out in the evaluation reports) were verified (for computational reproducibility), and all 3ie funded products (reports, data, and code files) were submitted to 3ie.

Simultaneously, we initiated a project to check the available datasets for computational reproducibility, as we believed this was the first step towards ensuring the reliability of evidence (Brown, Cameron, and Wood 2014). This project built upon our experience of conducting replications of external studies (non-3ie funded studies) as part of our Replication Windows (3ie, "Replication Studies", 2018).

\section{Sample}

Between 2008 and 2020, 179 impact evaluations had been completed. Our review of the datasets indicated that we had access to both data and statistical code for 133 out of the 179 closed studies (Figure 1). We were unable to reproduce the findings for the remaining 42 studies due to lack of data, statistical code, or both. Given that the availability of data and code is a prerequisite to testing the computational reproducibility of a study for the remainder of this paper, we focus on the 133 studies for which we had both data and code. $95 \%$ of these datasets were in Stata (multiple versions) and 3\% in R and Python. 


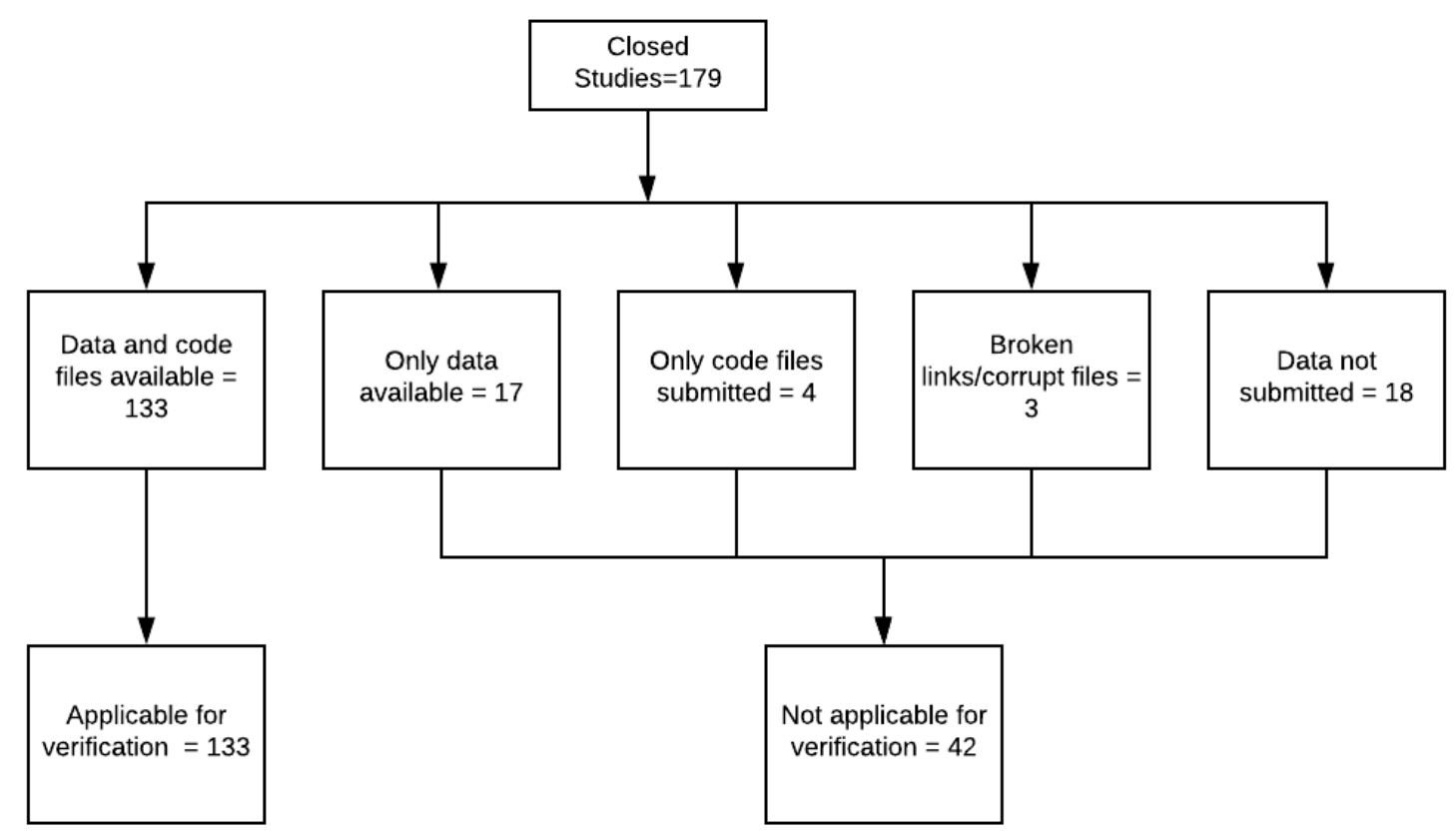

\section{Protocol}

Prior to describing the protocol, we first turn our attention to the definitions of the terms we use. The terms 'replication' and 'reproducibility' are often used interchangeably in the literature, and their use in this manner has been the subject of debate (Barba 2018). For the purposes of this paper, we use the term "computational reproducibility". We define this as the process whereby original data and statistical code (obtained from the authors) is used to verify whether the published/reported findings can be reproduced. In an earlier 3ie project, Wood, Muller, and Brown (2018), used the term 'push button replication' (PBR) to mean that once the data and code files are available, one can (potentially) push the button to reproduce reported findings. For this project, we used 
3ie's PBR protocol (Wood et.al. 2017) which was developed and tested in 3ie's Replication windows ${ }^{3}$. The protocol outlines a step-by-step procedure for conducting a push button replication, classification of studies on the basis of their reproducibility (see Table 1), and guidelines for comparing the results. However, while we largely followed the PBR protocol, we report two notable departures from the protocol: 1) The PBR protocol specifies main research results; 2) The PBR protocol also categorizes incomplete studies by a secondary classification, in order to classify the partially reproduced results. Given that our primary interest was only the verification of the computational reproducibility of all published results (rather than their replication), we did not follow these aspects of the protocol.

Table 1: Push Button Replication Classification

\begin{tabular}{|c|c|}
\hline Classification & Definition \\
\hline Comparable & Identical results or very small changes (like rounding) \\
\hline Minor differences & Small differences in coefficients and/or p-values \\
\hline Major differences & $\begin{array}{l}\text { Meaningful differences in reported outcomes (especially in the key } \\
\text { results) or the code does not reproduce published results }\end{array}$ \\
\hline No access & The original authors do not reply or decline to provide data or code \\
\hline Proprietary data & $\begin{array}{l}\text { Unable to provide data but provided code and Digital Subscriber } \\
\text { Line (DSL) documentation }\end{array}$ \\
\hline Incomplete & $\begin{array}{l}\text { Unable to reproduce part of the publication due to missing code } \\
\text { and/or data }\end{array}$ \\
\hline
\end{tabular}

\footnotetext{
${ }^{3}$ Read more about 3ie's replication program here: https://www.3ieimpact.org/evidence-hub/replication-studiesstatus
} 


\section{Process}

Our first step was to train a team of research assistants on the PBR process. Under the guidance of the team lead, this team was responsible for conducting the reproductions using the PBR protocol. Figure 2 describes the PBR process. On receiving data and code, we first screened for completeness of the files and checked for identifiers. The next step was to run and troubleshoot minor errors (for instance, changing the file path as necessary, or the use of different software versions, or the installation of add-on programs) the statistical code. Once the code ran smoothly, we compared the outputs with the published results. The study was then classified as indicated in Table 1. Metadata from each study was coded into an Excel sheet.

\section{Figure 2: Push Button Replication flow diagram}

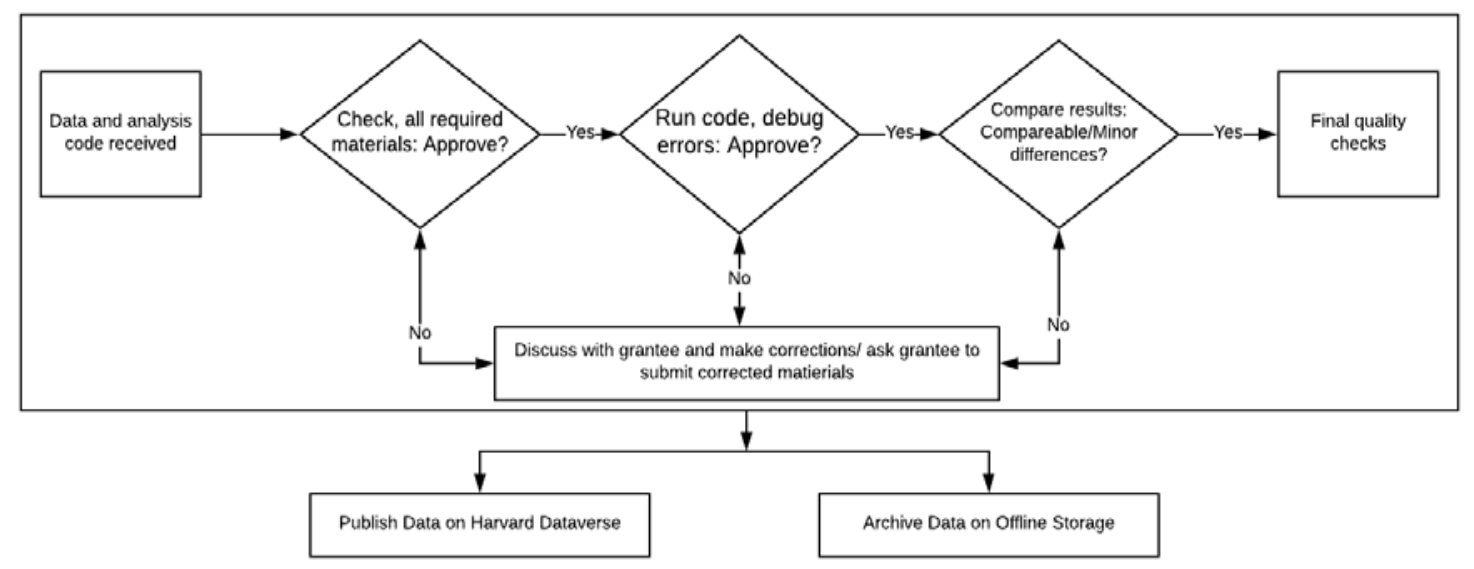

The next step entailed communicating with the study team (that conducted the evaluation) to share these results and request revisions (if necessary). For ongoing evaluations, we included the PBR feedback as part of our regular quality assurance 
processes. The original research team then re-submitted the revised report, data/code files and the PBR was conducted again. Over 2-3 submissions, we were able to resolve issues and most studies were classified as "comparable". However, the communication process was different in the case of older evaluations (those which closed in 2017 or earlier). In this case, despite repeated efforts to communicate our PBR findings, we often did not hear back from the teams. Thus, most of these datasets retained their original classification.

\section{Results}

\section{Push Button Replication classification}

For the 133 studies in our sample, in Figure 3 we display the classification and discuss the details below. We successfully reproduced results from 96 studies (72\%), i.e. the code ran on the data and produced comparable results to those presented in the final evaluation reports submitted to 3ie (identified as 'comparable studies' in the table). 24 studies (18\%) were categorized as incomplete i.e. we could only reproduce the results partially due to missing data/code, while another 13 studies (10\%) were categorized as having major differences. The issues in this category included differences in model specifications, variations in estimates (between reported findings and those generated by the data/code files) or statistical code not running. A2 Table (in the Appendix section) provides the details. 


\section{Figure 3: Reproducibility classification}

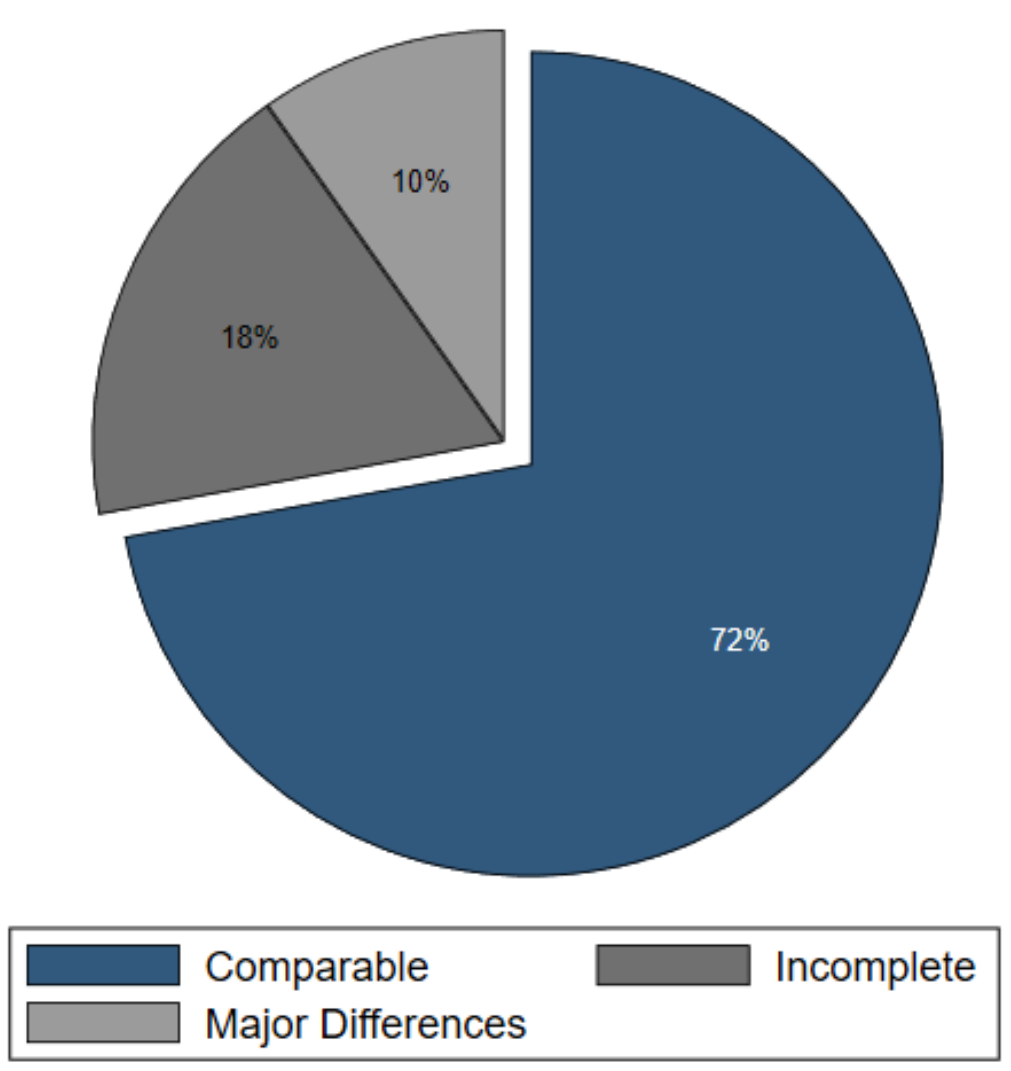

Source: Author's Calculation.

\section{Cumulative reproducibility rate}

Although we launched the first evidence programme in 2009 , studies only began to close in 2012 given the average 3-4-year duration of impact evaluations. Figure 4 maps the cumulative reproducibility rate (CRR), which we define as the proportion of studies (out of a total sample of 133 studies) classified as "comparable" in a given year. Between 2013 and 2015, the reproducibility rate consistently stayed below $40 \%$ even as the number of completed studies increased over the years. The reproducibility rate hovered around the 50\% mark for 2016 and 2017. However, since 2018, we note a dramatic increase in the reproducibility rate. 
Figure 4: Cumulative reproducibility rates over the years

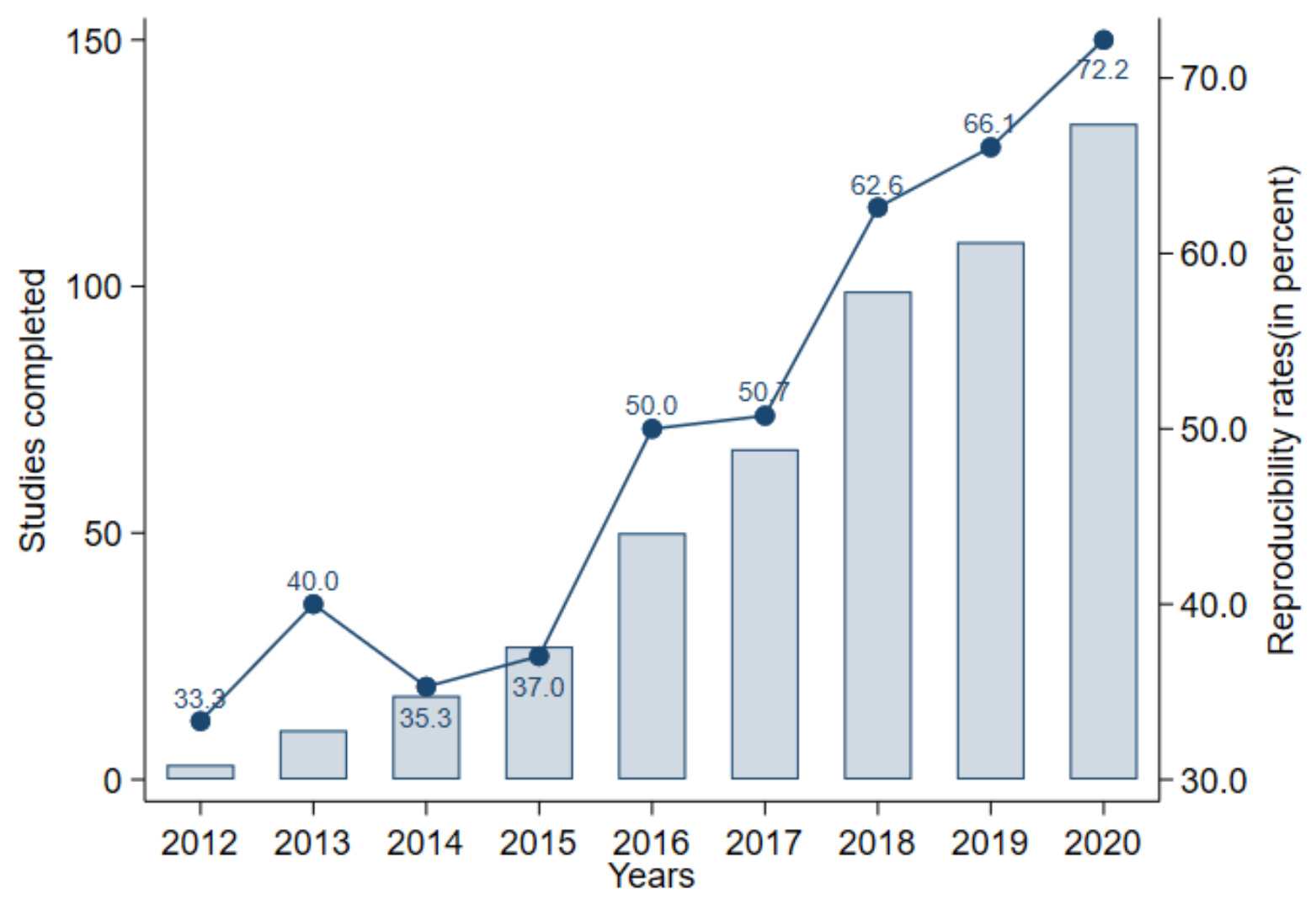

Source: Author's Calculation.

Figure 5 displays the classification of studies beginning from 2012, the year the first evaluation was completed. The number of comparable studies in 2018 is 28 which is much higher than the previous years. In 2018, no studies were categorized as having major differences, as all issues were resolved before publishing the data and code in the public domain. The subsequent years, namely 2019 and 2020, all studies were categorized as comparable. 
Figure 5: PBR classification over the years

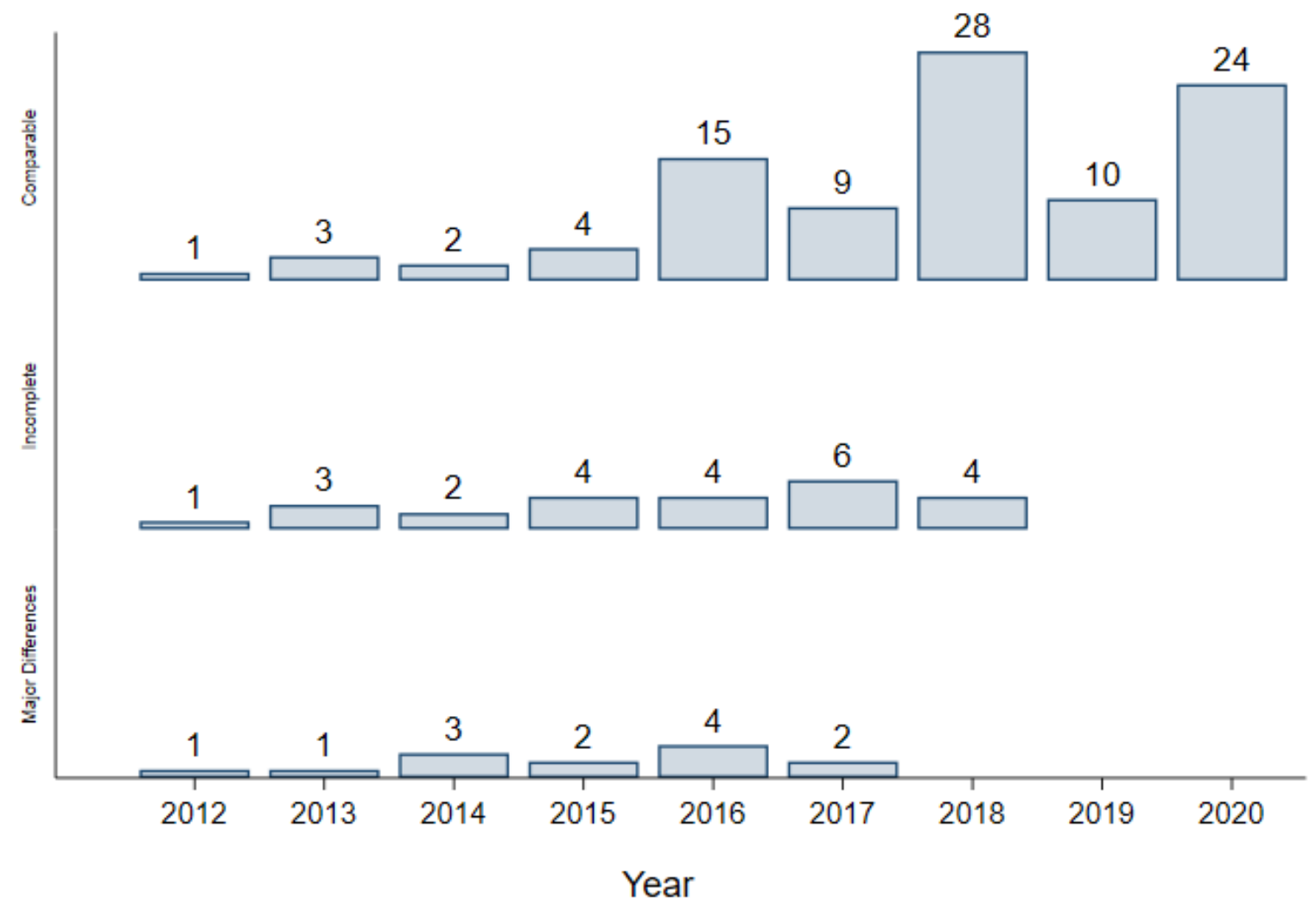

Source: Author's Calculation.

\section{Time use}

In Figure 6 we show that, on average, it took about 5 hours to complete the reproductions of one study (across multiple rounds). This is consistent with other studies such as Gertler, Galiani, and Romero (2018) who spent 4 hours on reproducing the studies in their sample. The time taken for comparable studies ranged from 2 to 30 hours based on the complexity of the analysis and the organization of the statistical code. Studies categorized as "incomplete" took between 1 and 8 hours. 
Figure 6: PBR classification by reproduction time

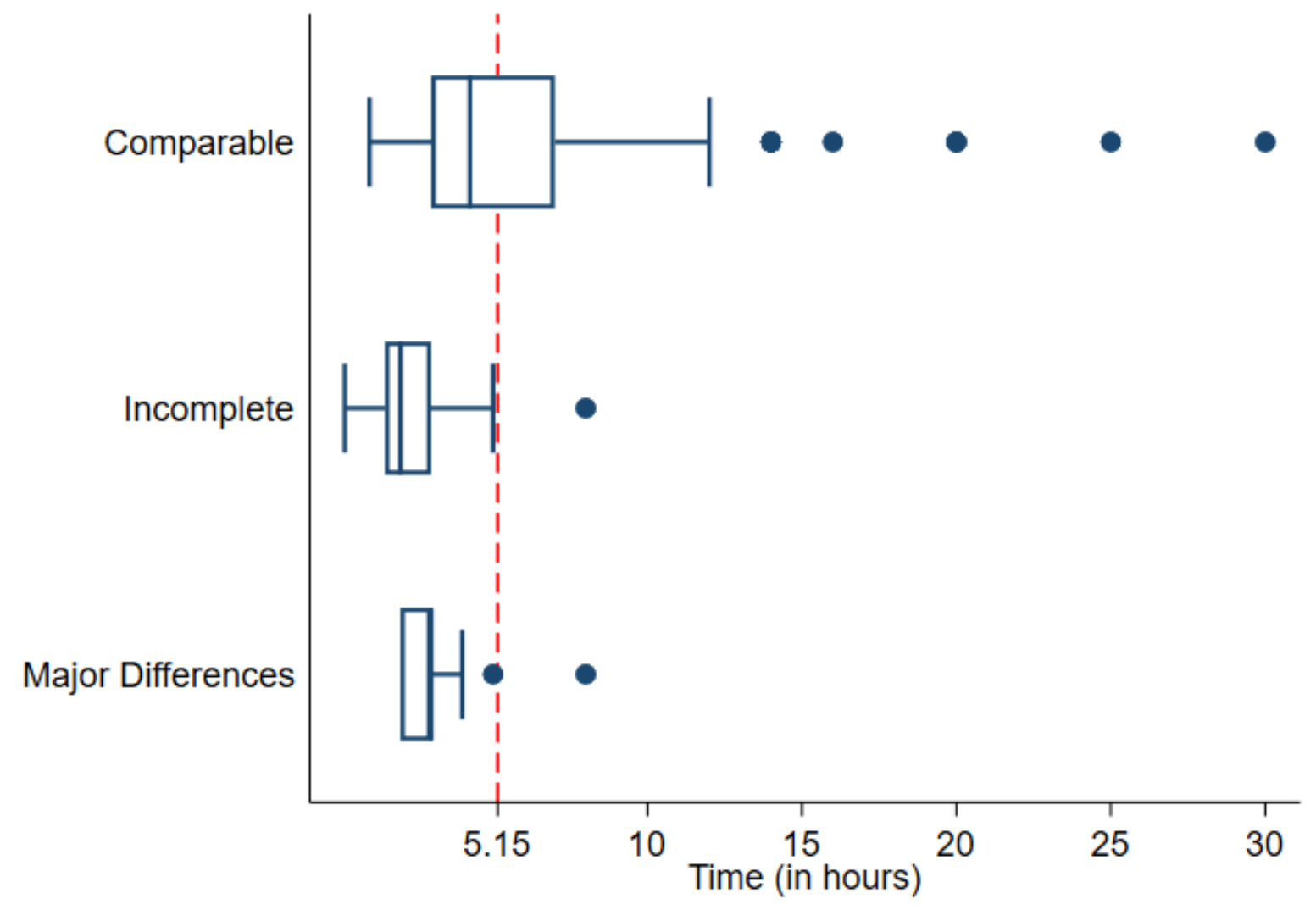

Source: Author's Calculation.

\section{Discussion}

Of our sample of 179 closed evaluations, we had access to 133 studies that had both data and code, which are a prerequisite for reproducing study results. Our primary finding is that we could reproduce findings for about three-fourths of the impact evaluations in our sample of these 133 studies. As noted, this sample excludes studies for which we do not have the requisite files (i.e., studies for which data, code or both are missing) for reproduction of results. Similar papers computing reproducibility rates have taken different approaches to the definition of their sample and of the denominator, and 
hence none of the studies are strictly comparable. However, we share some of the results from the literature for reader interest.

Wood, Muller, and Brown (2018) reported a reproducibility rate of $25 \%$ but has included studies for which data and/or codes had not been made available. Gertler, Galiani, and Romero (2018) reported a reproducibility rate of $37 \%$ for studies with analysis data and code, and $14 \%$ for studies with raw data, cleaning codes over and above analysis data and code.

Moving on to discuss the results of our project, in our view, there are two principal reasons for the relatively high reproducibility rate, especially since 2018 . First, the enforcement of our Research Transparency Policy (3ie, "Research Transparency Policy", 2018) was an important milestone, as final grant payments were only made once the PBR process was complete. As noted in our paper, our cumulative reproducibility rate was on average around $40 \%$ until 2018 when the policy was launched. As a direct consequence of the enforcement of this policy, the annual reproducibility rate for 2018 was $87.5 \%$ (inclusive of the studies that were completed between January- April 2018, prior to the implementation of the policy in April 2018), a substantially higher rate than previous years (53\% in 2017 and 65\% in 2016, as noted in A3 Figure). We find that for the years 2019 and 2020 we have a 100\% reproducibility rate which is also a direct effect of the grant payments only being made after the completion of the PBR. Second, unlike the other studies (Gertler, Galiani, and Romero 2018; Wood, Muller, and Brown 2018), we had previous associations with the original researchers in the form of past 3ie grantees. This helped us in resolving the issues over multiple iterations of the reproducibility process and resulted in a higher number of 
studies being classified as comparable. This is in contrast to other papers where there was only iteration.

As an institution dedicated to the use of rigorous evidence to inform development policies and programming, 3ie considers the reproducibility process as one tool we use to assure this rigor and the credibility of the evidence. Our finding that it is possible to improve reproducibility rates with a dedicated intervention is heartening. Oftentimes, as we have found in our work, the lack of verifiability of evaluation results may be due to human error (typographical or programming errors, for instance). But, in other instances, the inability to reproduce results could imply a larger problem including is organized outputs, teams not submitting all statistical files, or even providing outputs that are generated using different model specifications to the ones that had been previously reported.

Our key takeaway is that it is not enough to fund the generation of evidence - it is also our responsibility, as donors and researchers, to ensure the evidence is reliable prior to emphasizing its use. The issue is not limited to the reliability of the individual study, but rather that poor reliability has cumulative effects. 3ie produces and promotes the use of systematic reviews as a more reliable source of generalizable evidence for broader policy-making purposes than individual studies. This is because systematic reviews provide a complete and exhaustive summary of current rigorous evidence relevant to a research question and use standardized methods to collect secondary data, critically appraise research studies, and synthesize findings. This implies greater reliability on the collective evidence around a research topic. However, this reliability depends heavily on the reliability of the constituent studies. 
Based on our experiences in this project, we offer a few lessons we learned in the process of enforcing our research transparency policy and working on this reproducibility project:

Lesson \#1: Making data open data is an ongoing process. While 3ie was committed to open science right from its inception, our understanding of what is needed to make data transparent evolved over time. So, initially, we only requested datasets. But, gradually, as the awareness grew that datasets alone were not enough, we began requesting code files and other materials. Conducting this reproducibility exercise seemed to be the natural next step. Collaborators often mention that they have a lot of datasets available but that these data are not in a condition to be shared publically. Our suggestion is to start with sharing what is feasible - even one dataset and code, while simultaneously improving on research practices (such as writing reproducible code). As these practices become standard over a period of time, sharing data publically will become easier. Given the many advantages to sharing data, even small steps such as documenting all steps of data processing, explicitly reporting why transparent sharing is not possible, etc. can go a long way (Crüwell et. al., 2018).

\section{Lesson \#2: Policy articulation and enforcement can serve as important steps to} re-inforce commitment to transparency. Similar to our grant agreement, a number of journals have now begun to mandate the submission of data and code. Yet, without enforcement, the policies for open access may remain just that. For instance, Wood, Muller, and Brown (2018) and Gertler, Galiani, and Romero (2018) note that although journals required the submission of data and code, these files were not available. The 
enforcement may require the investment of dedicated resources to check that the data and code are submitted, and that the results replicate.

Lesson \#3: Open data begins with informed consent. While our grant contracts clearly indicate that data will be posted in the public domain, we found that, in a few instances, consent letters used by evaluation teams do not reflect this. In this scenario, it may be questionable ethically to publicly share even de-identified data. For studies where the consent process is complete, it may be best to contact the Institutional Review Board for approval. But, for new projects, we suggest the use of specific consent language. Institutions such as the Qualitative Data Repository offer sample templates (Qualitative Data Repository(QDR) 2019).

\section{Lesson \#4: Conduct reproducibility immediately after a project is complete.}

Turning now to learnings on PBR processes specifically, we find that it is best to check reproducibility immediately after the data analysis and publication process is complete. The reproducibility exercise is facilitated by good coding practices, version control, etc. But, conducting a reproducibility exercise sooner rather than later means that original study team members are still available to respond to queries and to address errors. This also means data can be posted in the public domain in a timely way. As noted earlier in our paper, our $100 \%$ reproducibility rate for the years 2019 and 2020 is largely due to this factor

Lesson \#5: Build internal capacity to manage reproductions. A surprise learning is that the PBR process can be completed within a span of 4-5 hours even by staff who may not be (initially) adept at the use of statistical software. Prior to conducting this reproducibility exercise, we provided our team of research assistants with basic training 
on Stata followed by a more focused training on the PBR process. This proved adequate for the purpose and staff gained experience of reviewing and writing good code. However, one challenge that emerged was the evaluation teams conduct their analyses using different software, such as Stata, R, etc. While Stata was the most common choice, we also had to be prepared to conduct reproductions in R. More recently, we have noted an increase in the diversity of software used (for instance, we have recently received datasets and code files in SPSS/SAS/Python).

The reproducibility project has helped 3ie strengthen its existing quality assurance processes and has introduced a number of new practices to improve openness practices. For instance, 3ie has streamlined its data submission and management practices with staff providing guidance to researchers on how to make their results reproducible. Additionally, 3ie has also begun to explore the curation, management and sharing of qualitative data, which has its own complexities.

\section{Limitations}

We note several limitations to our paper. First, our sample comprises impact evaluations funded by 3ie. Thus, the findings may not apply to all evaluations and cannot be generalized. However, we think our insights may be useful for other funders who may be interested in mandating higher standards in transparency of research they fund.

Second, our focus is on computational reproducibility using the PBR method. The biggest limitation to computational reproducibility is that it only verifies whether the code reproduces the results in the final evaluation reports submitted to 3ie. This process does 
not verify how the results are obtained or whether the hypothesis is legitimate or not. We cannot make any claims about the validity of the model used or any manipulations done to the data prior to analysis.

Lastly, we look at only the studies that have both data and code, which is a pre-requisite for computational reproducibility. One view could be that that the reproducibility estimates are inflated as we are not accounting for all the studies (i.e. studies with and without data and code) for computing the reproducibility rates. Our position is that since our focus was on identifying the reproducibility rates for studies where datasets and code files are available, we do not include studies where these are not available. However, in order to maintain the transparency of the sample, we have clearly indicated these exclusions in our paper.

\section{Conclusion}

The FAIR principles (launched in 2016) provide guidelines to make data findable, accessible, interoperable and reusable (Wilkinson et.al. 2016). Along with other institutions, 3ie is also committed to following these guidelines, while also working towards ensuring research transparency more broadly. Our Research Transparency policy defines our approach in this direction. Our vision is to ensure that the evidence we fund is verifiable so that it can be used to inform policies and programs.

In an effort to test the extent to which evidence is verifiable, we implemented a project to computationally reproduce findings from 133 studies. Our finding that only about three-fourths of the evaluations in our sample are replicable suggests that greater attention is needed to ensuring the reliability of evidence. In recent years, the Open 
Science movement has made great strides. For instance, a number of research donors have endorsed Plan $\mathrm{S}^{4}$ which is a recent initiative aimed at ensuring that evidence is publically accessible in a timely way. In a similar vein, some studies have found that incentivizing researchers (through the use of online badges (Open Science Framework 2019), for instance) has also increased the rate of data sharing (Rowhani-Farid, Allen, and Barnett 2017; Kidwell et.al 2016). These are important initiatives to ensuring that research processes are transparent and findings are easily accessible. But, evidence from evaluations is only credible when it can be verified. In our view, procedures to check reproducibility of evaluation and research findings are a necessary step towards ensuring the verifiability of evidence.

\section{Acknowledgements}

The authors would like to thank all the 3ie-funded evaluation teams who submitted data and code (especially in the cases when it was not contractually mandated) and worked with us patiently to ensure that the PBR process could be completed. We are also grateful to our team of 3ie research assistants who have worked hard to conduct PBRs.

\section{Disclosure statement}

We (authors) acknowledge that the data used in the analysis and assessment of the quality of evidence is sponsored by 3ie, which employed all the authors of the paper at the time of its writing.

${ }^{4}$ Available from: https://www.coalition-s.org/ 


\section{References}

1. Barba, LA. 2018. "Terminologies for reproducible research." arXiv preprint arXiv:1802.03311.

2. Brown, AN, DB Cameron, BD Wood. 2014 "Quality evidence for policymaking: I'll believe it when I see the replication." Journal of Development Effectiveness. 6(3):215-35.

3. Crüwell S, van Doorn J, Etz A, Makel MC, Moshontz H, Niebaum JC, et al. 2018. "7 Easy Steps to Open Science: An Annotated Reading List" [Internet]. PsyArXiv. Available from: psyarxiv.com/cfzyx

4. Galiani, S, P Gertler, M Romero. 2017. "Incentives for replication in economics." National Bureau of Economic Research.

5. Gertler P, S Galiani, M Romero. 2018. "How to make replication the norm" (vol 554, pg 417, 2018). Nature.;555(7698):580.

6. Humphreys M, De la Sierra RS, Van der Windt P. 2013. "Fishing, commitment, and communication: A proposal for comprehensive nonbinding research registration." Political Analysis. 21(1):1-20.

7. International Initiative for Impact Evaluation (3ie). 2018, "Replication Studies" [Internet]. [cited July 3, 2019]. Available from: https://www.3ieimpact.org/evidence$\underline{\text { hub/replication-studies-status }}$ 
8. International Initiative for Impact Evaluation (3ie). 2018. "3ie Research Transparency Policy" [Internet]. [cited July 1,2019]. Available from: https://www.3ieimpact.org/sites/default/files/2019-06/3ie-research-transparencypolicy.pdf

9. Ioannidis, JP. 2005. "Why most published research findings are false." PLoS medicine;2(8): e124.

10. Khatua, S. 2018. "What's the deal with Push Button Replication?." [cited July 1, 2019]. In: Evidence Matters [Internet]. Available from: https://www.3ieimpact.org/blogs/whats-deal-push-button-replications

11. Kidwell, MC, Lazarević LB, Baranski E, Hardwicke TE, Piechowski S, Falkenberg LS, Kennett C, Slowik A, Sonnleitner C, Hess-Holden C, Errington TM. 2016. "Badges to acknowledge open practices: A simple, low-cost, effective method for increasing transparency." PLoS biology. 14(5): e1002456.

12. Loken, E. 2019. "The replication crisis is good for science." [cited July 4, 2019]. In The Conversation [Internet] Available from: http://theconversation.com/thereplication-crisis-is-good-for-science-103736

13. Open Science Collaboration. 2015. "Estimating the reproducibility of psychological science." Science;349(6251):aac4716.

14. Open Science Framework (OSF). "Open Science Badges" [Internet]. [cited July 8, 2019]. Available from: https://cos.io/our-services/open-science-badges/

15. Plan S [Internet]. [cited July 1, 2019]. Available from: https://www.coalition-s.org/ 
16. Qualitative Data Repository (QDR). "Templates for Researchers" [Internet]. [cited July 1, 2019]. Available from: https://qdr.syr.edu/quidance/templates

17. Rowhani-Farid A, Allen M, Barnett AG. 2017. "What incentives increase data sharing in health and medical research? A systematic review." Research integrity and peer review.;2(1):4.

18. Simmons JP, Nelson LD, Simonsohn U. 2011 "False-positive psychology: Undisclosed flexibility in data collection and analysis allows presenting anything as significant". Psychological science;22(11):1359-66.

19. Wood BDK, Müller R, Brown AN. 2018. "Push button replication: Is impact evaluation evidence for international development verifiable?" PLoS ONE 13(12): e0209416. https://doi.org/10.1371/journal.pone.0209416

20. Wood BD, Brown AN, Djimeu E, Vasquez M, Yoon S, Burke J. 2017. "Replication Protocol for Push Button Replication (PBR)" [Internet]. [cited July 1, 2019]. Available from: https://www.3ieimpact.org/sites/default/files/201812/replication-protocol-pbr.pdf

21. Wilkinson, M. D. et al. (2016). "The FAIR Guiding Principles for scientific data management and stewardship". Scientific Data, 3, 160018. doi:10.1038/sdata.2016.18 


\section{Appendix}

A1 Table: Definitions for Ineligibility criteria

\begin{tabular}{|l|l|}
\hline Issue & Description \\
\hline Broken Link & $\begin{array}{l}\text { Often Researchers provide website links or cloud storage } \\
\text { links to their data and code. Overtime, these links become } \\
\text { inactive and it becomes impossible to retrieve the data. }\end{array}$ \\
\hline Only data & $\begin{array}{l}\text { A number of researchers only submitted data. Without the } \\
\text { codes accompanying the data a PBR cannot be conducted } \\
\text { and so result cannot be verified. }\end{array}$ \\
\hline Corrupt data files & $\begin{array}{l}\text { Data files are submitted by researchers in a number of file } \\
\text { formats. Overtime due multiple usage or even computer } \\
\text { viruses these files become corrupt and unusable. }\end{array}$ \\
\hline Data not submitted & $\begin{array}{l}\text { Researchers have outright refused or not submitted the data } \\
\text { even after following up. }\end{array}$ \\
\hline Only code files available & $\begin{array}{l}\text { Researchers have submitted code files but no data. As } \\
\text { result we cannot verify the studies. }\end{array}$ \\
\hline
\end{tabular}

A2 Table: Details of studies with Major Differences

\begin{tabular}{|l|c|}
\hline Issue & Number of studies \\
\hline Model specification and data disaggregation & 3 \\
\hline Statistical code not compiling & 2 \\
\hline Variations in the estimates & 6 \\
\hline
\end{tabular}




\begin{tabular}{|l|c|}
\hline Poorly annotated code files & 2 \\
\hline Total number of studies & 13 \\
\hline
\end{tabular}

A3 Figure: Yearly reproducibility rate

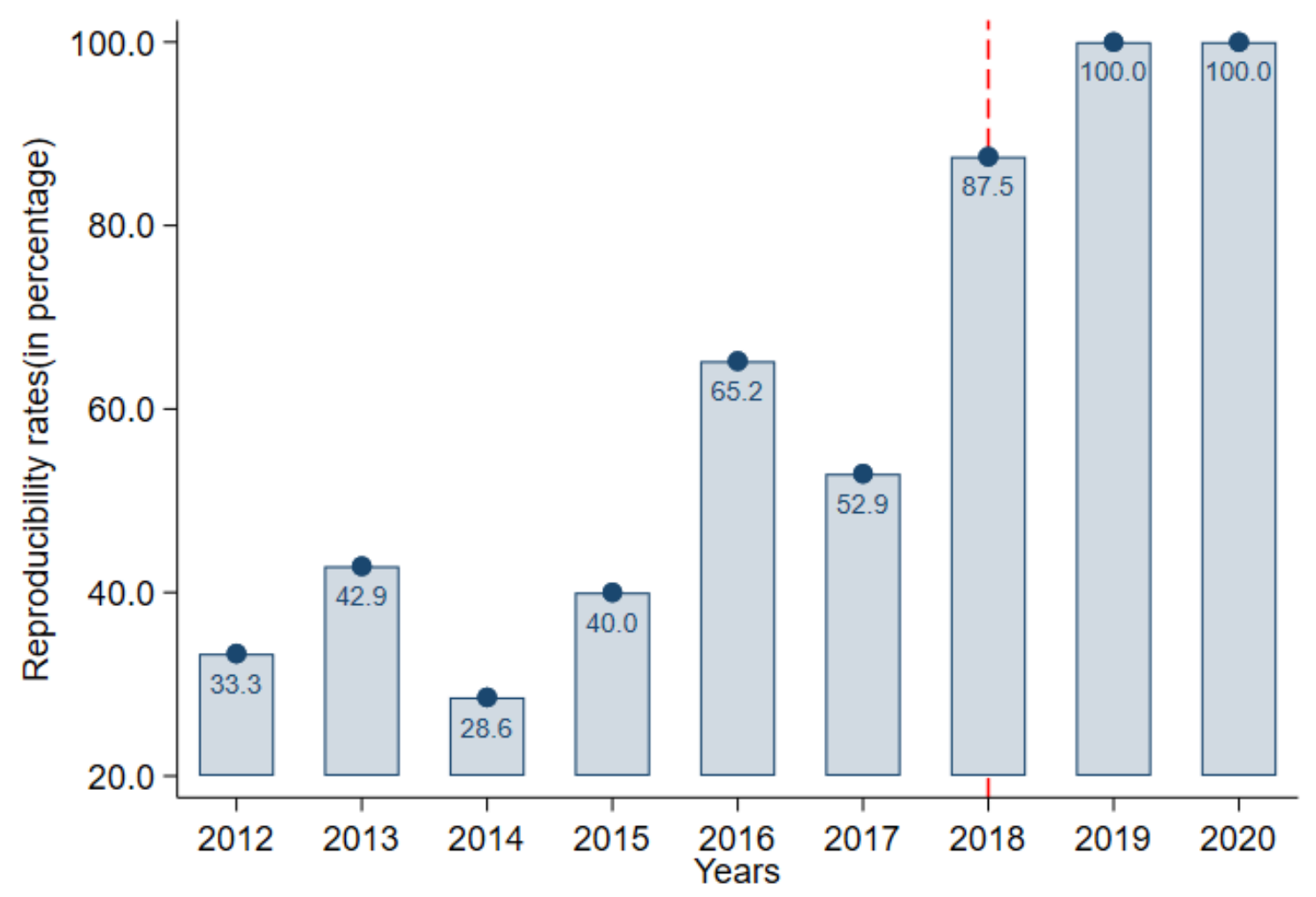

Source: Author's Calculation. Policy was implemented in 2018 